\title{
Pathogenic variants in KPTN gene identified by clinical whole-genome sequencing
}

\author{
Isabelle Thiffault, ${ }^{1,2,3}$ Andrea Atherton, ${ }^{4}$ Bryce A. Heese, ${ }^{4}$ Ahmed T. Abdelmoity, ${ }^{4}$ \\ Kailash Pawar, ${ }^{4}$ Emily Farrow, ${ }^{1,3,4}$ Lee Zellmer, ${ }^{1}$ Neil Miller, ${ }^{1}$ Sarah Soden, ${ }^{1,3,4}$ \\ and Carol Saunders ${ }^{1,2,3}$ \\ ${ }^{1}$ Center for Pediatric Genomic Medicine, Children's Mercy Hospital, Kansas City, Missouri 64108, USA; \\ ${ }^{2}$ Department of Pathology and Laboratory Medicine, Children's Mercy Hospitals, Kansas City, Missouri 64108 , \\ USA; ${ }^{3}$ University of Missouri-Kansas City School of Medicine, Kansas City, Missouri 64108, USA; ${ }^{4}$ Department \\ of Pediatrics, Children's Mercy Hospitals, Kansas City, Missouri 64108, USA
}

Corresponding author: csaunders@cmh.edu

(c) 2020 Thiffault et al. This article is distributed under the terms of the Creative Commons

Attribution-NonCommercial License, which permits reuse and redistribution, except for commercial purposes, provided that the original author and source are credited.

Ontology terms: complex febrile seizures; delayed fine motor development; delayed gross motor development; epileptic encephalopathy; intellectual disability, severe; macrocephaly at birth; polymorphic focal epileptiform discharges

Published by Cold Spring Harbor Laboratory Press

doi:10.1101/mcs.a003970
Abstract Status epilepticus is not rare in critically ill intensive care unit patients, but its diagnosis is often delayed or missed. The mortality for convulsive status epilepticus is dependent on the underlying aetiologies and the age of the patients and thus varies from study to study. In this context, effective molecular diagnosis in a pediatric patient with a genetically heterogeneous phenotype is essential. Homozygous or compound heterozygous variants in KPTN have been recently associated with a syndrome typified by macrocephaly, neurodevelopmental delay, and seizures. We describe a comprehensive investigation of a 9-yr-old male patient who was admitted to the intensive care unit, with focal epilepsy, static encephalopathy, autism spectrum disorder, and macrocephaly of unknown etiology, who died of status epilepticus. Clinical whole-genome sequencing revealed compound heterozygous variants in the KPTN gene. The first variant is a previously characterized 18-bp in-frame duplication (c.714_731dup) in exon 8, resulting in the protein change p.Met241_Gln246dup. The second variant, c.394 +1G > A, affects the splice junction of exon 3. These results are consistent with a diagnosis of autosomal recessive KPTN-related disease. This is the fourth clinical report for KPTN deficiency, providing further evidence of a wider range of severity.

[Supplemental material is available for this article.]

\section{INTRODUCTION}

In recent years, next-generation sequencing (NGS) technologies have revolutionized approaches in clinical genetics. Whole-exome sequencing (WES) or whole-genome sequencing (WGS) allows diagnoses in many patients with complex phenotypes and unusual clinical presentations. As the cost of NGS falls, it has become feasible to use this powerful technology in clinical care, simultaneously unraveling variations in about 19,000 genes. Technological advances have led to the ability to sequence, analyze, and interpret entire genome data in a timely manner, clearly changing the diagnostic paradigm and proving to be cost-effective in many cases (Soden et al. 2014). Clinical diagnostic sequencing currently focuses on identifying causal mutations in the exome ( $1 \%$ of the genome), where most disease-causing mutations are known to occur. WGS permits analysis of coding regions as well as regulatory elements that control gene expression; however, noncoding variants 
remained largely unexplored in clinical diagnostics because of the interpretive challenges (Warman Chardon et al. 2015). Although it lacks the depth of coverage of an exome, WGS can have more reliable and uniform sequence coverage, particularly in regions of the genome with low sequence complexity or high GC-rich content. Both WGS and WES approaches have benefits and limitations, but the limitation of NGS gene panels and WES should be considered prior to clinical testing (Williams et al. 2008; Soden et al. 2014; Ankala et al. 2015; Warman Chardon et al. 2015). In this context, the present work demonstrates the application of clinical WGS in the pediatric population in which we provided a fast, accurate, and cost-effective molecular diagnosis in a pediatric patient with a genetically heterogeneous phenotype.

\section{RESULTS}

\section{Clinical Presentation}

A 9-yr-old Caucasian male was admitted to the Children's Mercy Hospital pediatric intensive care unit (PICU) for status epilepticus and further evaluation of a suspected underlying genetic condition because of his history of macrocephaly, intractable epilepsy, autism, severe developmental delays, hypotonia, and hypoglycemia. Dysmorphic features include frontal bossing, sunken eye sockets, downslanting palpebral fissures, small ears, thin upper lip, and small nose (Fig. 1). The prenatal history was unremarkable: Delivery was at full term, and neonatal development was normal. He was the third child for his parents together, born to a 26-yr-old mother and 30-yr-old father. Family history was noncontributory. At birth, he weighed 2.71 kilograms and was $49.5 \mathrm{~cm}$ long. His head circumference was noted to be
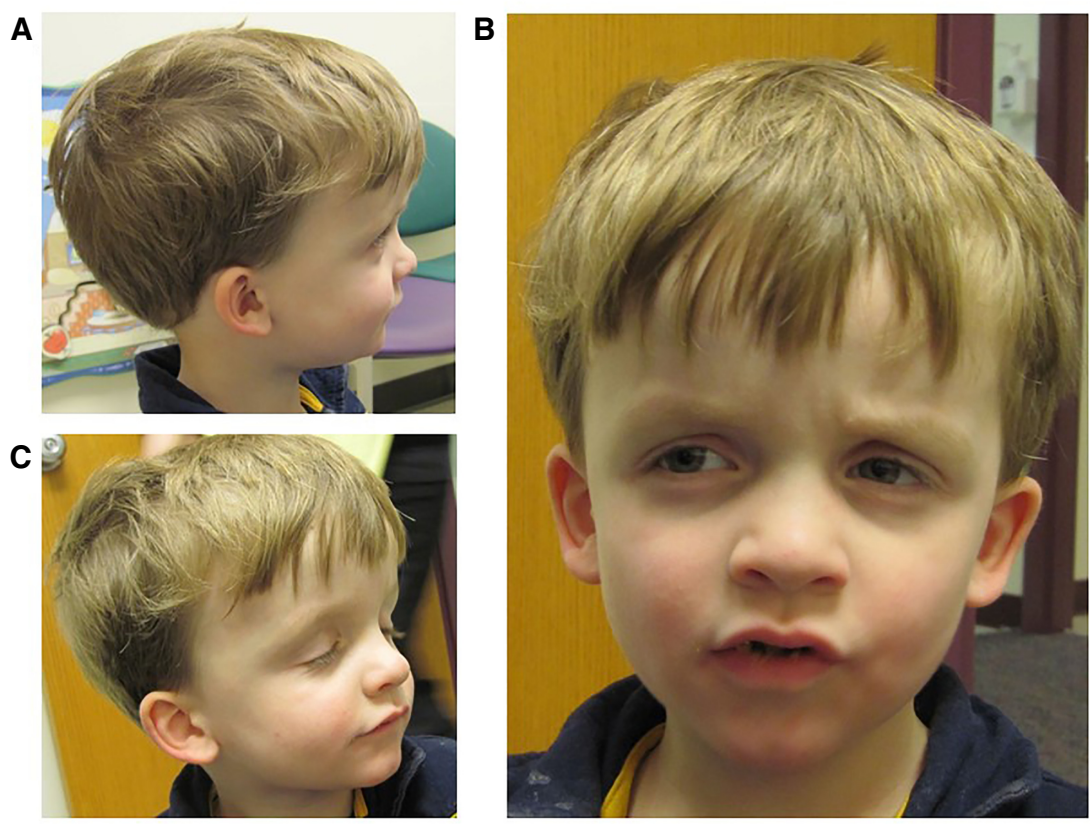

Figure 1. Our patient was a 9-yr-old male with epilepsy, static encephalopathy, autism spectrum disorder, and (A) macrocephaly of unknown etiology who died of status epilepticus. (B) Several facial dysmorphisms were found, including frontal bossing, sunken eye sockets, downslanting palpebral fissures, small ears, thin upper lip, and small nose. (C) At 4 yr old, he was normal height and weight, but his head circumference measured $57 \mathrm{~cm}$ (>98th percentile). 
greater than the 99th percentile at birth. At $4 \mathrm{yr}$ old, he was of average height and weight, but his head circumference measured $57 \mathrm{~cm}$ ( $>98$ th percentile, +4SD). He had poor tracking and eye contact but a normal ophthalmologic exam. A head CT was completed at 2 mo of age and reported to be normal. At 3 mo of age, he started to have partial seizures but EEG was normal. At 5 mo of age he was noted to have hepatosplenomegaly accompanied by hypoglycemic episodes. Developmentally, he smiled socially at $2 \mathrm{mo}$, rolled at $8 \mathrm{mo}$, sat without assistance at 10-11 mo, crawled at $14 \mathrm{mo}$, and walked at $2.5 \mathrm{yr}$. A brain MRI was normal at 4 mo of age; however a second, performed at $9 \mathrm{yr}$ of age, was concerning for a possible arteriovenous malformation, with abnormal hyperintense T2/FLAIR signals in the subcortical white matter of the right anteromedial temporal lobe. An extensive etiologic workup was normal, including high-resolution karyotype, CGH microarray, PTEN sequencing, fragile $\mathrm{X}$, enzyme analysis for Gaucher, mucopolysaccharidosis I and II, plasma amino acids, pristanic and phytanic acids, very long chain fatty acid (VLCFA), and urine organic acids. Mild elevations of C14 species on an acylcarnitine profile in 2010 were detected in conjunction with ketotic hypoglycemia and were likely associated with physiologic response to fasting. Previously, the seizures occurred at a frequency of 1-2 per hour, consisting of focal tonicclonic seizures involving his left or right side of the body but occasionally progressed to status epilepticus, and a pentobarbital infusion was started with the goal to acquire burst suppression. Unfortunately, this was unsuccessful, and he passed away at age 9.

\section{Genetic Analysis}

Clinical WGS was performed on the patient and WES was performed on his healthy mother, following informed consent. The patient was compound heterozygous for two pathogenic variants in the KPTN gene (Table 1). This gene was ranked 351st in the Phenomizer gene list. The first variant identified was an 18-bp in-frame duplication (c.714_731dup ACCGACCACATCTGCAGA; rs587777148) in exon 8, resulting in the protein change p.Met241_GIn246dup. This variant has been previously reported in trans with a second truncating variant in multiple affected individuals from two Amish families (Baple et al. 2014). Prior in vitro transfection studies indicated the mutant protein is mislocalized and accumulates in neurons of affected individuals, leading to a dominant negative effect (Baple et al. 2014). The p.Met241_Gln246dup variant is not found in population databases such as dbSNP, Exome Variant Database, or ExAC but was found in 1 of 560 Amish control chromosomes (Baple et al. 2014); 136/275574 in gnomAD data set (0.05\%). The second variant, c.394 + 1G > A, affects the splice junction of exon 3 . This variant has not been reported in affected individuals but is predicted to cause aberrant splicing. This variant was observed in $\sim 0.01 \%$ individuals of European ancestry in the NHLBI Exome Sequencing Project and Exome Aggregation Consortium (ExAC); 19/277090 in gnomAD data set (0.007\%). The c.394 + 1G > A variant, but not the c.714_731dup variant, was inherited from the mother, suggesting these variants are in trans. This genotype was confirmed by Sanger sequencing and is consistent with a diagnosis of autosomal recessive KPTN-related disease. In addition, two variants of unknown significance were reported ((CHD2-NM_001271.3:c.5268G > C (p.Gln1756His); CTDP1-NM_004715.4:c.1219T > C (p.Trp407Arg)). No incidental findings were reported for this case. The turnaround time was $33 \mathrm{~d}$.

\section{DISCUSSION}

The use of NGS techniques by clinical laboratories has risen tremendously and has greatly facilitated the elucidation of the etiologic diagnosis in patients suspected of having a genetic disease. The various approaches, including WES, WGS, and targeted panels, all have benefits and limitations. Targeted panels offer a specific list of genes relevant to the clinical 


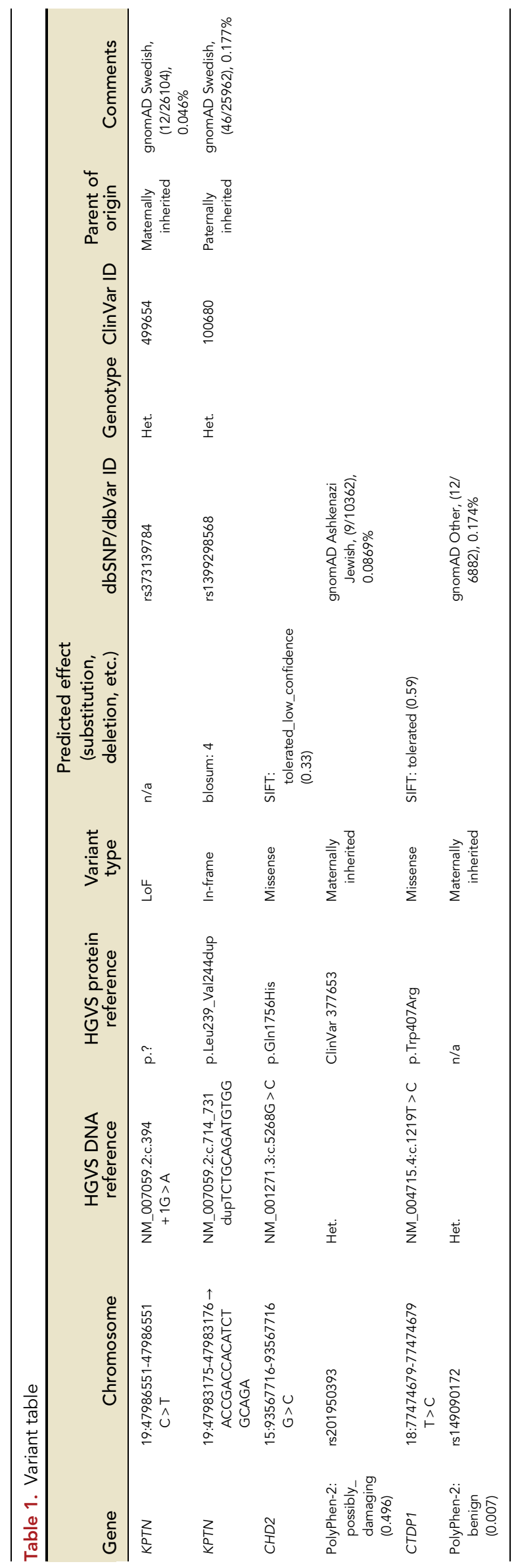


phenotype in question and often boast higher coverage than WES/WGS. However, whether this improves sensitivity, particularly in Mendelian disorders, is not clear. In addition, a wide range of interlaboratory variability exists for gene lists offered for the same condition. For instance, commercial clinical NGS panels for macrocephaly include anywhere from 12 to 44 genes. Although targeted panels may appear to be a more economical approach to WES/ WGS, this is only the case if the gene relevant to the patient being tested is present on the panel, which is often difficult to know a priori, particularly in patients with nonspecific symptoms such as intellectual disability or seizures. In many cases, serial testing of additional genes and panels quickly surpasses the expense of WES/WGS (Soden et al. 2014). In addition, targeted panels may fail to incorporate newly discovered disease-associated genes. For example, new genes associated with both syndromic and nonsyndromic macrocephaly have been identified, further expanding the genetic heterogeneity to more than 165 conditions, including 17 metabolic disorders, associated with macrocephaly (Williams et al. 2008; Keppler-Noreuil et al. 2014; Koh et al. 2014; Marchese et al. 2014; Sugathan et al. 2014; Nevado et al. 2015; Nguyen et al. 2015). One such gene, KPTN, encoding kaptin, was first reported in January of 2014 in patients with a syndrome typified by macrocephaly, neurodevelopmental delay, and seizures (MRT41; OMIM\# 615637) (Baple et al. 2014; Pajusalu et al. 2015). KPTN is not currently offered in any clinical panels for macrocephaly in the United States. Of the clinical laboratories listed in the NextGxDx website, only two of 16 offer KPTN as part of an autism/intellectual disability panel, and only two of 17 include it as part of an epilepsy panel. The comparison of such gene lists is difficult for clinicians and the curation is onerous for the clinical laboratory to manage. Clinical WES/WGS removes the guesswork as far as which gene to include, as all genes relevant to the patient's phenotype are queried in the analysis process. In the current case, by using clinical WGS in a 9-yr-old Caucasian male admitted in pediatric intensive care unit for status epilepticus, a diagnosis of KPTN-related disease was made.

A comparison of the individual clinical signs in patients reported with KPTN-related disease are shown in Table 2 . The most unifying features are macrocephaly (92\%), developmental delay (100\%), and intellectual disability (100\%). Recurrent dysmorphic features included frontal bossing, abnormal head shape, and prominent chin. With the exception of the p.Met241_Gln246dup, which is predicted to be nonfunctional by producing a misfolded altered protein, all patients have truncating variants (p.S259*; p.S223Qfs*50; p.S200Ifs*55); no patients were homozygous for the p.Met241_Gln246dup. RT-PCR or western blot experiments were not performed to assess if those variants result in nonsense-mediated mRNA decay or whether a truncated protein (lacking the carboxy-terminal amino acids) is produced. The second variant uncovered in our patient, c.394+1G >A, is located upstream of the two previously reported truncating variants. This is the first splicing variant reported in KPTN, it is likely to result in nonsense-mediated mRNA decay, which may explain the more severe outcome. Additional external factors such as modifier genes could also influence the phenotype. Of the 12 previous patients described, seven have survived into their second and third decades, with two having expired at ages 29 and 30 to a head injury and pneumonia (Table 2; Baple et al. 2014; Pajusalu et al. 2015; Lucena et al. 2020). At this time, limited data exist for genotype-phenotype correlations; further phenotyping and natural history of disease study are needed to understand the clinical spectrum and prognosis of KPTNrelated syndrome.

This is the fourth clinical report for KPTN-deficiency, the 13th patient but the first to die in childhood with status epilepticus, providing further evidence of a wider range of severity.

Several studies have shed new light on the molecular and cellular processes that orchestrate the human neuronal circuitry and is defective in neurological disorders (Ropers et al. 2011; Riviere et al. 2012; Baple et al. 2014; Koh et al. 2014; Sugathan et al. 2014). 


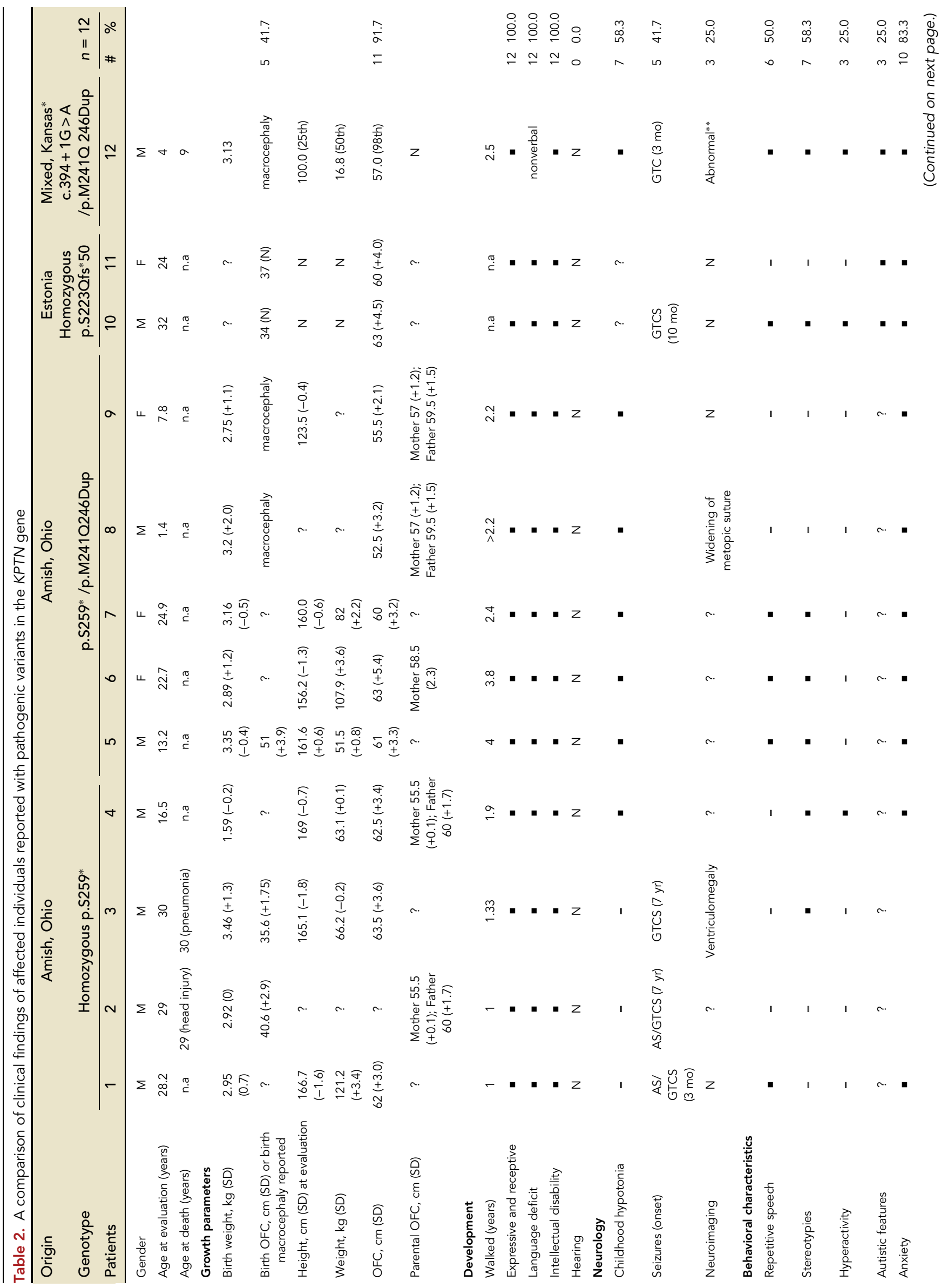




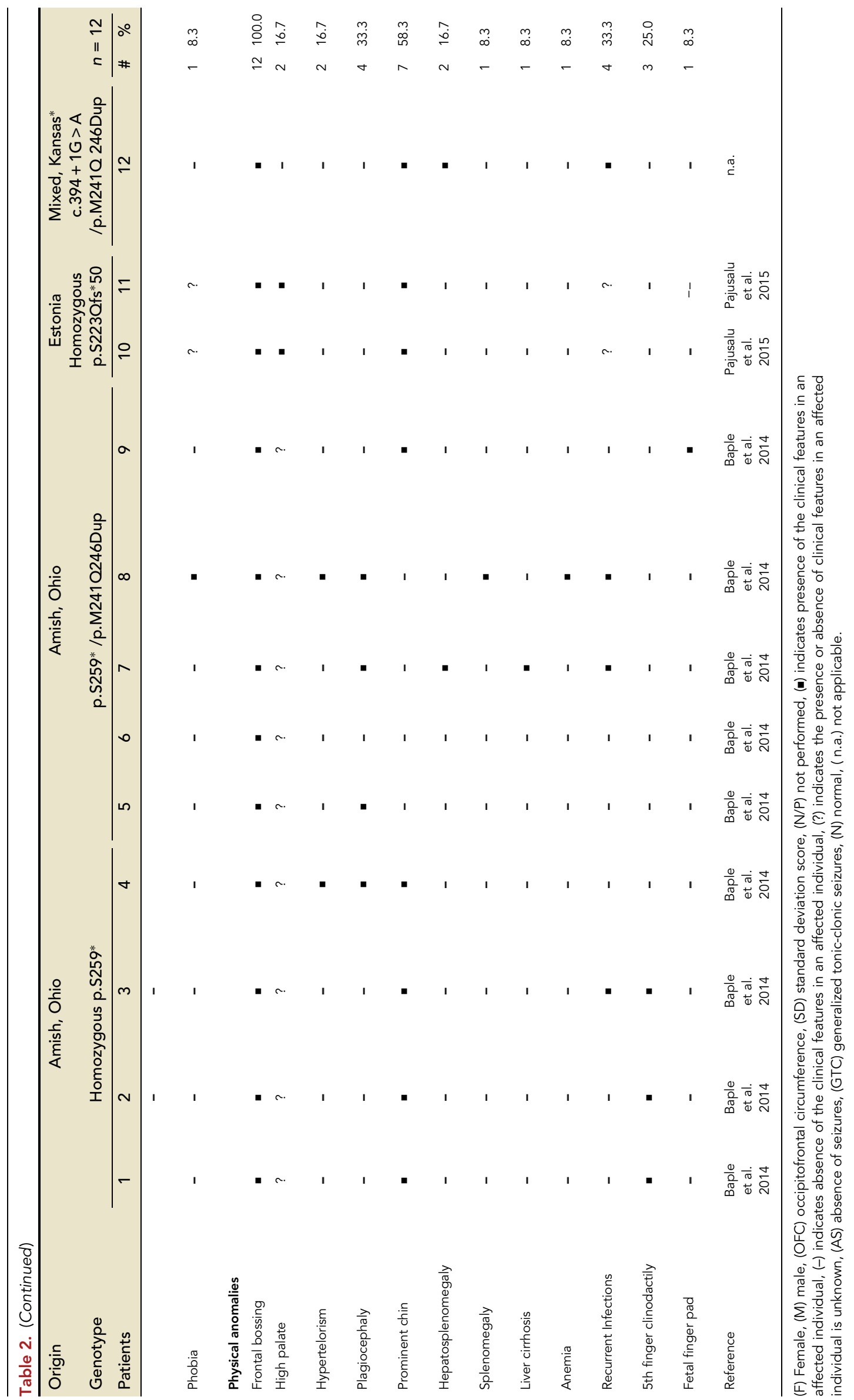


Evidence implicating defects in proteins involved in actin dynamics, microtubule homeostasis, and vesicle trafficking in developmental disorders via effects on neuronal development and migration and brain architecture has accumulated over the past decade. The role of microtubule homeostasis (LIS1 and DCX) or tubulin (TUBA1A, TUBA8, TUBB2A, TUBB4A, TUBB2B, TUBB3, and TUBB and TBCE) genes has been established in several brain disorders. More recently, the actin-encoding genes ACTB and ACTG1 have been shown to be involved in brain malformations causing Baraitser-Winter syndrome (OMIM \# 243310) (Riviere et al. 2012). Additional intellectual disability genes involved in actin dynamics and vesicle trafficking include STXBP1 and SYP (Barcia et al. 2013; Kato 2015). Patients with KPTN deficiency consistently exhibit macrocephaly, intellectual disability, and developmental delay. Furthermore, KPTN is a novel actin binding protein, enriched in neuronal growth cones and cortical sites of neurons at early developmental stages, likely playing a central role in modulating neuron morphology and growth. Although genes affecting many of these pathways are associated with hearing loss, to date, no patients with KTPN-related disease, including our patient, have been reported with deafness. As with several other published studies (Riviere et al. 2012; Soden et al. 2014; Ankala et al. 2015; Warman Chardon et al. 2015; Willig et al. 2015), WGS was found to be a rapid and cost-efficient approach for molecular diagnostic of a genetically heterogeneous condition (Thiffault et al. 2019). Thus, this report confirms the occurrence of KPTN-related syndrome outside of the Amish population and demonstrates the variability in the phenotypic spectrum and severity. Pathological mechanisms of abnormal neuronal actin cytoskeleton and discrepancy between the underlying phenotypes caused by KPTN deficiency remain to be elucidated.

\section{METHODS}

Genomic DNA was extracted from peripheral blood mononuclear cells using a Chemagen MSM1 robot (PerkinElmer). WGS was prepared using the Kapa Hyper library prep omitting PCR. Sequencing was completed on an Illumina HiSeq 2500 instrument utilizing paired end $2 \times 125$ base pair reads with 44 Chemistry (Illumina). The proband's sample was sequenced to a depth of $115.16 \mathrm{~Gb}$ for a mean coverage of $\sim 37 \times$ (Supplemental Table S1). Bidirectional sequence was assembled, aligned to reference gene sequences based on human genome build GRCh37/UCSC hg19, and analyzed using custom-developed software, RUNES, and VIKING (Saunders et al. 2012; Soden et al. 2014; Willig et al. 2015). Variants were filtered to a $1 \%$ minor allele frequency and then prioritized by the American College of Medical Genetics (ACMG) categorization (Richards et al. 2015), OMIM identity, and phenotypic assessment. Alignments were viewed using Integrative Genomic Viewer software version 2.3.8 (IGV; Broad Institute). A candidate gene list was generated by Phenomizer using Human Phenotype Ontology (Kohler et al. 2014) terms: Macrocephaly (HPO:0000256), Muscular hypotonia (HPO:0001252), Autism (HPO:0000717), Seizures (HPO:0001250), Global developmental delay (HPO:0001263), and Abnormal facial shape (HPO: 0001999), with a cutoff at $P$-value of 0.5 . This gene list contained approximately 700 genes and was imported into VIKING to guide the analysis; however, a separate analysis, not limited by this gene list, was performed in parallel to look for relevant pathogenic genotypes in genes not included in the HPO gene list. Pathogenic, likely pathogenic, and variants of unknown significance in HPO genes were reported; likely benign and benign variants are not reported but are made available upon request. Incidental findings in the 56 genes recommended by ACMG were requested by the family and analyzed for pathogenic and likely pathogenic variants only. 
Competing Interest Statement The authors have declared no competing interest.

\section{Referees}

Sander Pajusalu

Anonymous

Received January 7, 2019; accepted in revised form April 16, 2020.

\section{ADDITIONAL INFORMATION}

\section{Data Deposition and Access}

Our patient consent does not permit patient sequence data to be uploaded to a data repository. The variants reported have been deposited in the ClinVar database (https://www.ncbi .nlm.nih.gov/clinvar/) and can be found under accession numbers VCV000100680.3, VCV000499654.1, and VCV000377653.5.

\section{Ethics Statement}

Written informed consent was obtained from the patient's legal guardians for publication of this case report and governed by the Institutional Review Board of Children's Mercy Hospitals and Clinics. A copy of the written consent is available for review by the Editorin-Chief of this journal.

\section{Acknowledgments}

We are very grateful to this family for allowing this case to be published. We thank our colleagues in the Center for Pediatric Genomic Medicine and Children's Mercy Hospital in Kansas City. This work was not supported by a grant.

\section{Author Contributions}

C.J.S., E.F., I.T., L.Z., and S.S. conceived and designed the experiments; C.J.S., E.F., and I.T. performed the experiments; N.M. and E.F. contributed reagents/materials/analysis tools; I.T. and C.J.S. wrote the paper; E.F., S.S., L.Z., B.H., A.A., and N.M. reviewed the manuscript; K.P. reviewed the manuscript and contributed to the clinical investigation of the patient; and A.A., B.H., and A.T.A. contributed to the recruitment and clinical investigations of the patient for the study.

\section{REFERENCES}

Ankala A, da Silva C, Gualandi F, Ferlini A, Bean LJ, Collins C, Tanner AK, Hegde MR. 2015. A comprehensive genomic approach for neuromuscular diseases gives a high diagnostic yield. Ann Neurol 77: 206-214. doi:10.1002/ana.24303

Baple EL, Maroofian R, Chioza BA, Izadi M, Cross HE, Al-Turki S, Barwick K, Skrzypiec A, Pawlak R, Wagner K, et al. 2014. Mutations in KPTN cause macrocephaly, neurodevelopmental delay, and seizures. Am J Hum Genet 94: 87-94. doi:10.1016/j.ajhg.2013.10.001

Barcia G, Barnerias C, Rio M, Siquier-Pernet K, Desguerre I, Colleaux L, Munnich A, Rotig A, Nabbout R. 2013. A novel mutation in STXBP1 causing epileptic encephalopathy (late onset infantile spasms) with partial respiratory chain complex IV deficiency. Eur J Med Genet 56: 683-685. doi:10.1016/j.ejmg.2013.09.013

Kato M. 2015. Genotype-phenotype correlation in neuronal migration disorders and cortical dysplasias. Front Neurosci 9: 181. doi:10.3389/fnins.2015.00181

Keppler-Noreuil KM, Sapp JC, Lindhurst MJ, Parker VE, Blumhorst C, Darling T, Tosi LL, Huson SM, Whitehouse RW, Jakkula E, et al. 2014. Clinical delineation and natural history of the PIK3CA-related overgrowth spectrum. Am J Med Genet A 164A: 1713-1733. doi:10.1002/ajmg.a.36552

Koh JY, Lim JS, Byun HR, Yoo MH. 2014. Abnormalities in the zinc-metalloprotease-BDNF axis may contribute to megalencephaly and cortical hyperconnectivity in young autism spectrum disorder patients. Mol Brain 7: 64. doi:10.1186/s13041-014-0064-z

Kohler S, Doelken SC, Mungall CJ, Bauer S, Firth HV, Bailleul-Forestier I, Black GC, Brown DL, Brudno M, Campbell J, et al. 2014. The Human Phenotype Ontology project: linking molecular biology and disease through phenotype data. Nucleic Acids Res 42: D966-D974. doi:10.1093/nar/gkt1026

Lucena PH, Armani-Franceschi G, Bispo-Torres AC, Bandeira ID, Lucena MFG, Maldonado I, Veiga MF, Miguel D, Lucena R. 2020. KPTN gene homozygous variant-related syndrome in the northeast of Brazil: a case report. Am J Med Genet A 182: 762-767. doi:10.1002/ajmg.a.61492 
Marchese M, Conti V, Valvo G, Moro F, Muratori F, Tancredi R, Santorelli FM, Guerrini R, Sicca F. 2014. Autismepilepsy phenotype with macrocephaly suggests PTEN, but not GLIALCAM, genetic screening. BMCMed Genet 15: 26. doi:10.1186/1471-2350-15-26

Nevado J, Rosenfeld JA, Mena R, Palomares-Bralo M, Vallespin E, Angeles Mori M, Tenorio JA, Gripp KW Denenberg E, Del Campo M, et al. 2015. PIAS4 is associated with macro/microcephaly in the novel interstitial 19p13.3 microdeletion/microduplication syndrome. Eur J Hum Genet doi:10.1038/ejhg.2015.51

Nguyen LS, Schneider T, Rio M, Moutton S, Siquier-Pernet K, Verny F, Boddaert N, Desguerre I, Munich A, Rosa JL, et al. 2015. A nonsense variant in HERC1 is associated with intellectual disability, megalencephaly, thick corpus callosum and cerebellar atrophy. Eur J Hum Genet doi:10.1038/ejhg.2015.140

Pajusalu S, Reimand T, Ounap K. 2015. Novel homozygous mutation in KPTN gene causing a familial intellectual disability-macrocephaly syndrome. Am J Med Genet A 167A: 1913-1915. doi:10.1002/ajmg.a.37105

Richards S, Aziz N, Bale S, Bick D, Das S, Gastier-Foster J, Grody WW, Hegde M, Lyon E, Spector E, et al. 2015. Standards and guidelines for the interpretation of sequence variants: a joint consensus recommendation of the American College of Medical Genetics and Genomics and the Association for Molecular Pathology. Genet Med 17: 405-424. doi:10.1038/gim.2015.30

Riviere JB, van Bon BW, Hoischen A, Kholmanskikh SS, O'Roak BJ, Gilissen C, Sullivan CT, Christian SL, AbdulRahman OA, Atkin JF, et al. 2012. De novo mutations in the actin genes ACTB and ACTG1 cause BaraitserWinter syndrome. Nat Genet 44: 440-444. doi:10.1038/ng.1091

Ropers F, Derivery E, Hu H, Garshasbi M, Karbasiyan M, Herold M, Nürnberg G, Ullmann R, Gautreau A, Sperling K, et al. 2011. Identification of a novel candidate gene for non-syndromic autosomal recessive intellectual disability: the WASH complex member SWIP. Hum Mol Genet 20: 2585-2590. doi:10.1093/ hmg/ddr158

Saunders CJ, Miller NA, Soden SE, Dinwiddie DL, Noll A, Alnadi NA, Andraws N, Patterson ML, Krivohlavek LA, Fellis J, et al. 2012. Rapid whole-genome sequencing for genetic disease diagnosis in neonatal intensive care units. Sci Transl Med 4: 154ra135. doi:10.1126/scitranslmed.3004041

Soden SE, Saunders CJ, Willig LK, Farrow EG, Smith LD, Petrikin JE, LePichon JB, Miller NA, Thiffault I, Dinwiddie DL, et al. 2014. Effectiveness of exome and genome sequencing guided by acuity of illness for diagnosis of neurodevelopmental disorders. Sci Transl Med 6: 265ra168. doi:10.1126/scitranslmed .3010076

Sugathan A, Biagioli M, Golzio C, Erdin S, Blumenthal I, Manavalan P, Ragavendran A, Brand H, Lucente D, Miles J, et al. 2014. CHD8 regulates neurodevelopmental pathways associated with autism spectrum disorder in neural progenitors. Proc Natl Acad Sci 111: E4468-E4477. doi:10.1073/pnas.1405266111

Thiffault I, Farrow E, Zellmer L, Berrios C, Miller N, Gibson M, Caylor R, Jenkins J, Faller D, Soden S, et al. 2019. Clinical genome sequencing in an unbiased pediatric cohort. Genet Med 21: 303-310. doi:10.1038/ s41436-018-0075-8

Warman Chardon J, Beaulieu C, Hartley T, Boycott KM, Dyment DA. 2015. Axons to exons: the molecular diagnosis of rare neurological diseases by next-generation sequencing. Curr Neurol Neurosci Rep 15: 64 doi:10.1007/s11910-015-0584-7

Williams CA, Dagli A, Battaglia A. 2008. Genetic disorders associated with macrocephaly. Am J Med Genet A 146A: 2023-2037. doi:10.1002/ajmg.a.32434.

Willig LK, Petrikin JE, Smith LD, Saunders CJ, Thiffault I, Miller NA, Soden SE, Cakici JA, Herd SM, Twist G, et al. 2015. Whole-genome sequencing for identification of Mendelian disorders in critically ill infants: a retrospective analysis of diagnostic and clinical findings. Lancet Respir Med 3: 377-387. doi:10.1016/ S2213-2600(15)00139-3 


\section{COLD SPRING HARBOR Molecular Case Studies}

\section{Pathogenic variants in KPTN gene identified by clinical whole-genome sequencing}

Isabelle Thiffault, Andrea Atherton, Bryce A. Heese, et al.

Cold Spring Harb Mol Case Stud 2020, 6: a003970 originally published online May 1, 2020

Access the most recent version at doi: $10.1101 / \mathrm{mcs} . a 003970$
Supplementary http://molecularcasestudies.cshlp.org/content/suppl/2020/05/21/mcs.a003970.D Material C1

References This article cites 22 articles, 3 of which can be accessed free at: http://molecularcasestudies.cshlp.org/content/6/3/a003970.full.html\#ref-list-1

License This article is distributed under the terms of the Creative Commons Attribution-NonCommercial License, which permits reuse and redistribution, except for commercial purposes, provided that the original author and source are credited.

Email Alerting Receive free email alerts when new articles cite this article - sign up in the box at the Service top right corner of the article or click here. 